\title{
Roles of programmed cell death protein 5 in inflammation and cancer (Review)
}

\author{
WEI WANG, XIAO-WEN SONG and CHENG-HAI ZHAO \\ Department of Pathophysiology, College of Basic Medical Science, \\ China Medical University, Shenyang, Liaoning 110122, P.R. China
}

Received July 4, 2016; Accepted September 19, 2016

DOI: $10.3892 /$ ijo.2016.3706

\begin{abstract}
PDCD5 (programmed cell death 5) is an apoptosis related gene cloned in 1999 from a human leukemic cell line. PDCD5 protein containing 125 amino acid (aa) residues sharing significant homology to the corresponding proteins of species. Decreased expression of PDCD5 has been found in many human tumors, including breast, gastric cancer, astrocytic glioma, chronic myelogenous leukemia and hepatocellular carcinoma. In recent years, increased number of studies have shown the functions and mechanisms of PDCD5 protein in cancer cells, such as paraptosis, cell cycle and immunoregulation. In the present review, we provide a comprehensive review on the role of PDCD5 in cancer tissues and cells. This review summarizes the recent studies of the roles of PDCD5 in inflammation and cancer. We mainly focus on discoveries related to molecular mechanisms of PDCD5 protein. We also discuss some discrepancies between the current studies. Overall, the current available data will open new perspectives for a better understanding of PDCD5 in cancer.
\end{abstract}

\section{Contents}

1. Introduction

2. Properties of PDCD5

3. PDCD5 and inflammation

4. Antitumor function of PDCD5

5. The antitumor mechanisms of PDCD5

6. Future perspectives

Correspondence to: Professor Cheng-Hai Zhao, Department of Pathophysiology, College of Basic Medical Science, China Medical University, Shenyang, Liaoning 110122, P.R. China

E-mail: cmu_chenghai@163.com

Key words: programmed cell death 5, inflammation, cancer, p53, P-glycoprotein

\section{Introduction}

Programmed cell death 5 (PDCD5), also designated TF-1 cell apoptosis-related gene-19 (TFAR19), is an apoptosis-related gene cloned in 1999 from the human leukemic cell line TF-1 (accession number AF014955 in GenBank) (1). Human PDCD5 gene is located on chromosome 19q12-q13.1 (2), and the integrated PDCD5 protein contains 125 amino acid (aa) residues (3). The wide expression pattern of PDCD5 protein in various cell lines indicated that it is a regulator in pathological and physiological processed (4). Decreased expression of PDCD5 has been found in many human tumors, including breast (5), gastric cancer (6), astrocytic glioma (7), chronic myelogenous leukemia (8) and hepatocellular carcinoma (9) (Figs. 1 and 2). Previous studies also showed that PDCD5 is involved in paraptosis (10), cell cycle regulation (11), ischemia/ reperfusion (4), immunoregulation (12) and viral infection (13).

However, the molecular mechanism of PDCD5 regulation during inflammation and cancer is very complex. According to the published literature, PDCD5 is essential for inflammation and cancer through regulating apoptosis. The present review is structured to provide a comprehensive overview of the functions and the mechanisms of PDCD5 in inflammation and cancer.

\section{Properties of PDCD5}

PDCD5 protein containing 125 amino acid (aa) residues shares significant homology with the corresponding proteins of species ranging from yeast to mice (1) (Fig. 3). Human PDCD5 protein can be divided into three structural regions: a rigid core region (residues 41-101 aa), $\mathrm{C}$-terminal residues (102-125 aa) and $\mathrm{N}$-terminal residues (3-40 aa) (3) (Fig. 4). In the study of Liu et al (14), it was found that the sequence region of PDCD5 ( $\mathrm{Asp}^{3}-\mathrm{Ala}^{19}$ ) contains four leucine residues, three alanine residues, and two polar amino acid residues by using NMR methods. They also found that PDCD5 without N-terminal residues significantly attenuates the apoptosis-promoting effects on myeloblastic leukemia HL-60 cells (14). The C-terminal region of PDCD5 ( $\mathrm{Val}^{109}-\mathrm{Val}^{116}$ ) includes four basic residues, which mediate the ability of the protein to bind to heparin, is important for PDCD5 to translocate through plasma membranes (15). The nuclear translocation of 
N口RMAL

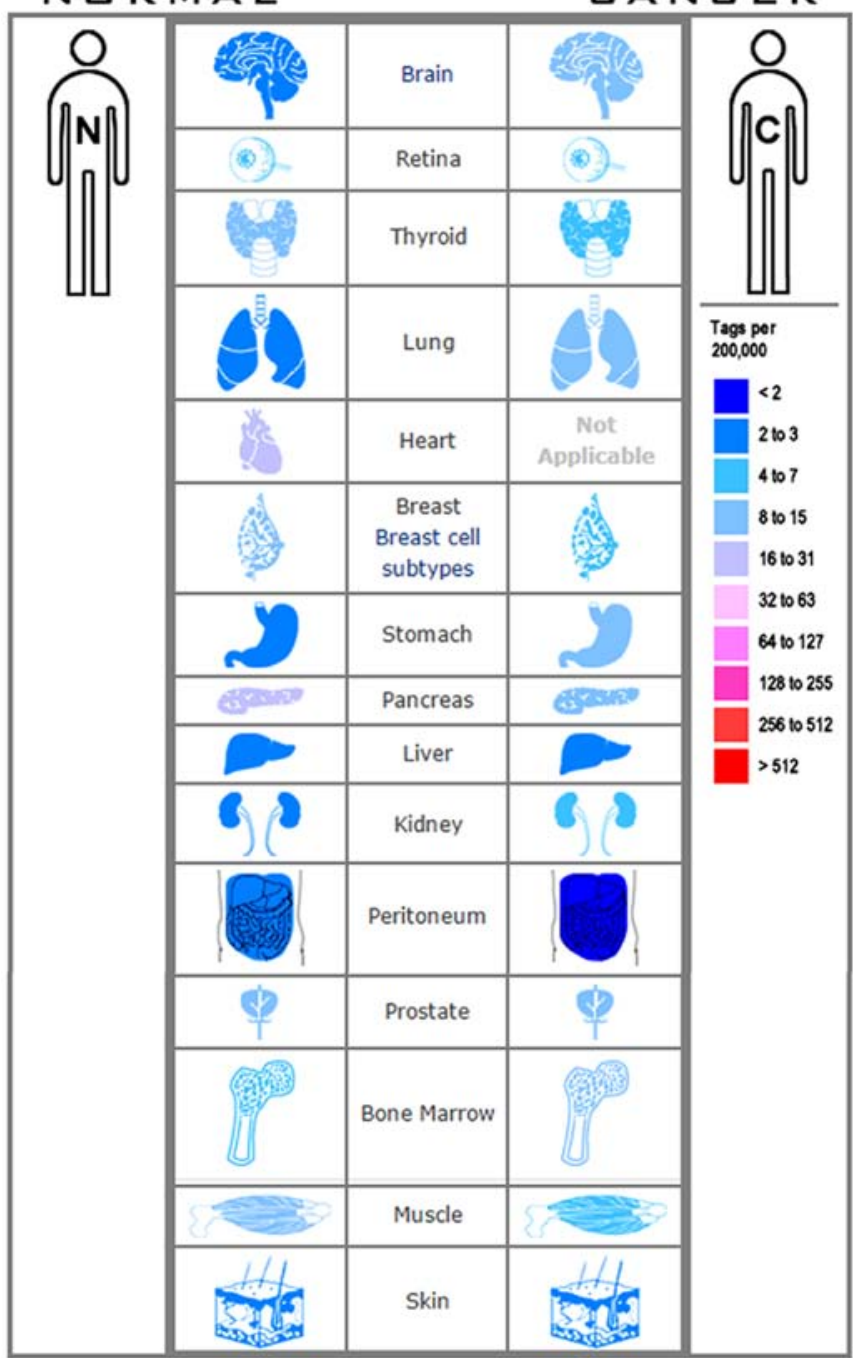

Figure 1. Expression profile for PDCD5 in human cancers.

PDCD5 is an early event of the apoptotic process, and may be a novel early marker for apoptosis (16).

\section{PDCD5 and inflammation}

Osteoarthritis (OA) is one of the most common chronic health conditions and a leading cause of pain and disability among adults (17). The main cause of OA is that articular chondrocytes lose proliferative capacity while maintaining the ability to produce proinflammatory mediators and matrix degrading enzymes (18). Cheng et al (19) found the enhancement of PDCD5 expression in OA cartilage compared with that in normal healthy cartilage. In the study of Yi et al (20) they also found that the level of PDCD5 was negatively correlated with the rate of chondrocyte apoptosis. These results indicated that PDCD5 is involved in the pathogenesis of OA.

PDCD5 also plays an important role in rheumatoid arthritis. Rheumatoid arthritis (RA) is a chronic systemic inflammatory joint disease characterized by hyperplasia of synovial tissue, inflammatory infiltrates and a progressive destruction of cartilage and bone (21). PDCD5 expression was

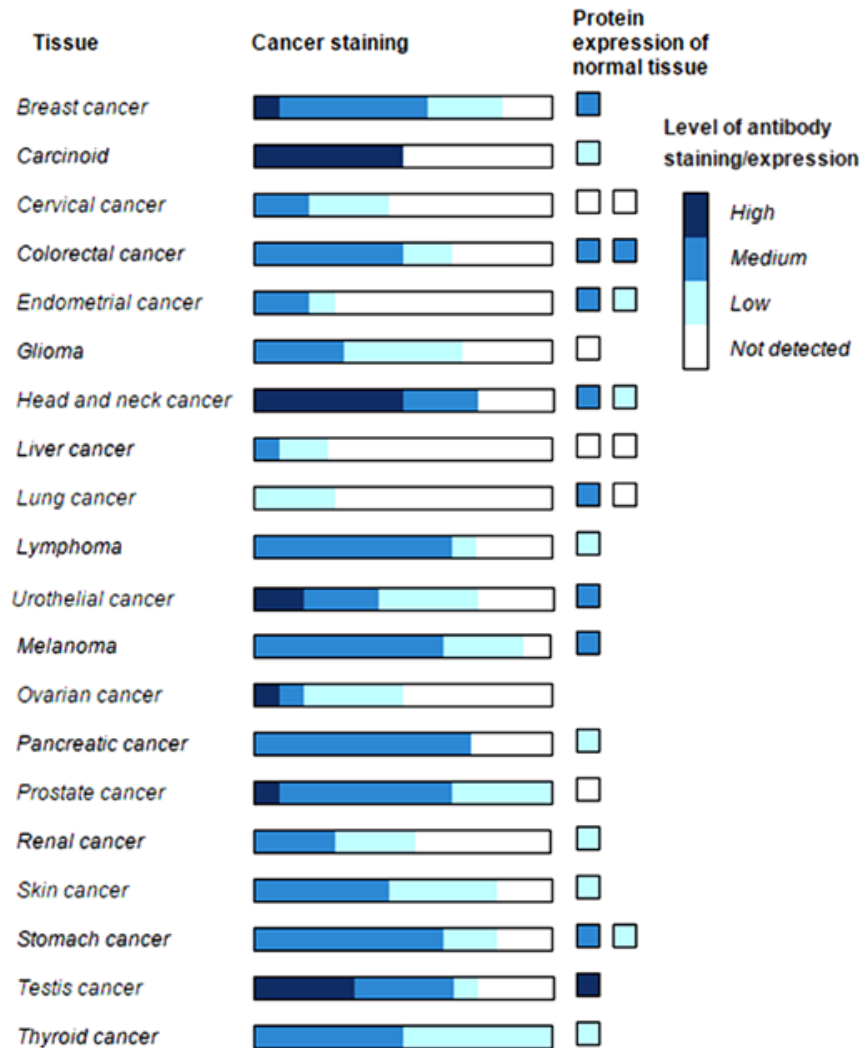

Figure 2. Ideograph of PDCD5 protein was generated by using Peptide Atlas.

low in RA patient-derived synovial tissue and cultured fibroblast-like synoviocytes (22). Plasma and synovial fluid PDCD5 abnormal expression and dysfunction may be correlated to tumor necrosis factor- $\alpha$ (TNF- $\alpha$ ) in RA patients (23). PDCD5 level was also negatively correlated with proinflammatory cytokine interleukin (IL)-17 levels both in serum and synovial fluid of RA patients (24). These results indicated that plasma and synovial fluid PDCD5 could be useful for monitoring the activity and progression of RA. Xiao et al (25) found that overexpression of PDCD5 increased the level of Foxp3 protein and percentage of Foxp $3^{+}$regulatory $\mathrm{T}$ (Treg) cells, and suppressed Th17 and Th1 responses in PDCD5 transgenic mice. A recent study showed that recombinant human PDCD5 (rhPDCD5) protein has prophylactic and therapeutic properties in a mouse model of multiple sclerosis by inhibiting Th1/Th17 differentiation and inducing apoptosis of predominantly pathogenic T cells (13).

The results of the present studies confirmed that PDCD5 serves as a guardian of immunological functions and that the PDCD5-FOXP3-Treg axis may be a therapeutic target for inflammation.

\section{Antitumor function of PDCD5}

Hematological oncology. PDCD5 is apoptosis-related gene originally cloned in 1999 from TF-1 human leukemic cell line undergoing apoptosis (1). From then on, PDCD5 was demonstrated to promote apoptosis in cancer cells in many studies. Ruan et al (8) found that PDCD5 mRNA was lower in both acute myeloid leukemia (AML) and chronic myeloid leukemia 

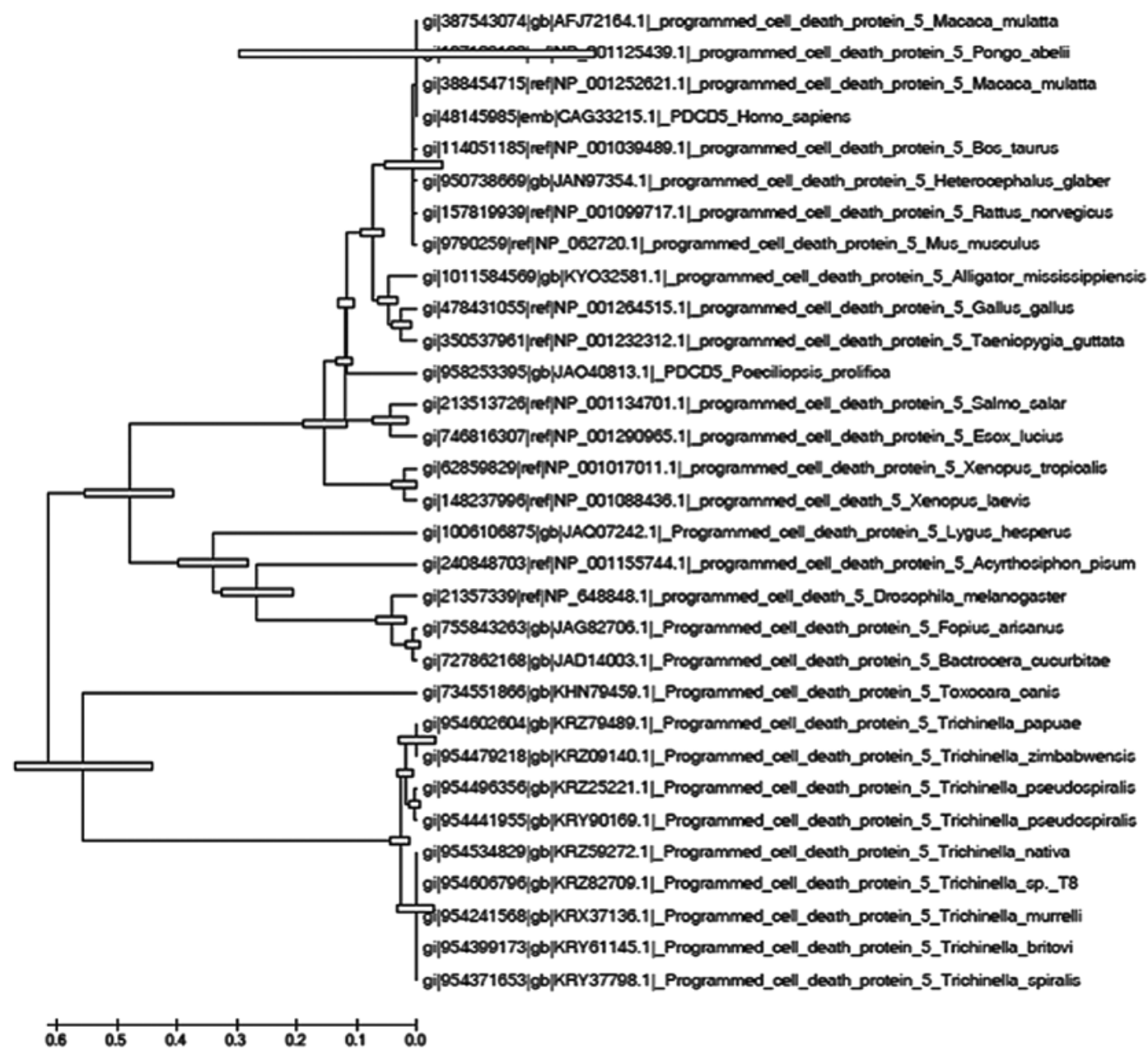

Figure 3. Multiple sequence alignments of PDCD5 by using the MEGA 5.1 software.
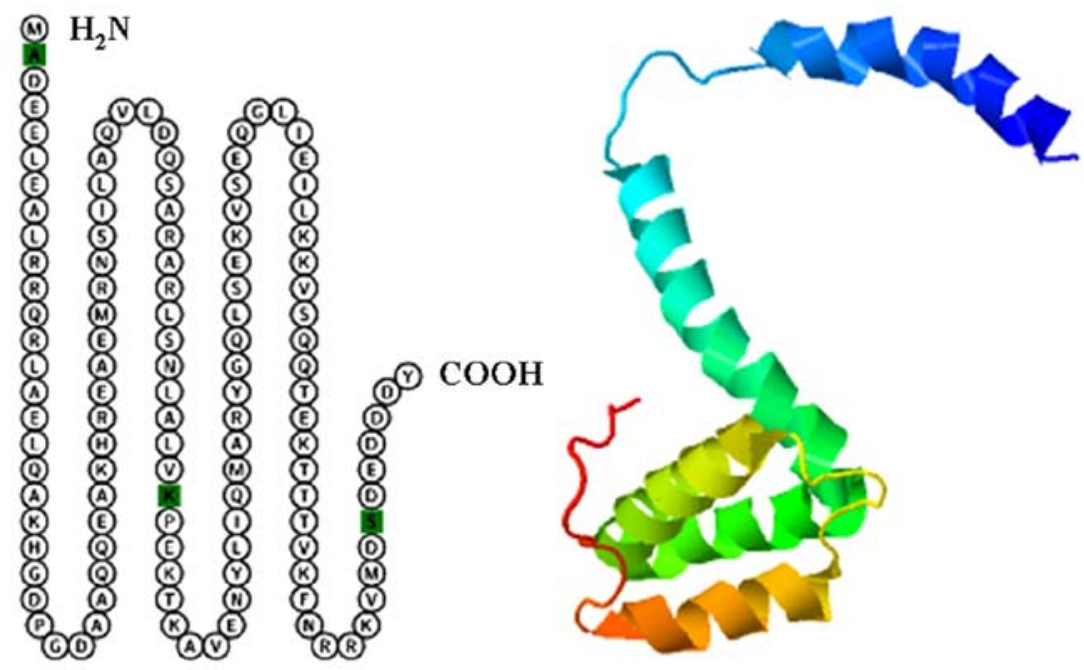

Figure 4. Structure of PDCD5 protein in RCSB Protein Data Bank.

(CML) marrow cells than that in normal donor marrow cells. They also observed a synergistic effect on apoptotic cell death in human CML K562 cells after combination therapy with adenovirus-mediated PDCD5 and idarubicin in vitro and in vivo (26). Shi et al (27) found that rhPDCD5 protein sensi- tizes K562 cells to apoptosis induced by chemotherapeutic drugs in vitro and in vivo.

Bone tumor. Osteosarcoma is the most frequent malignant bone tumor (28). Zhao et al (30) found that adenovirus-delivered 


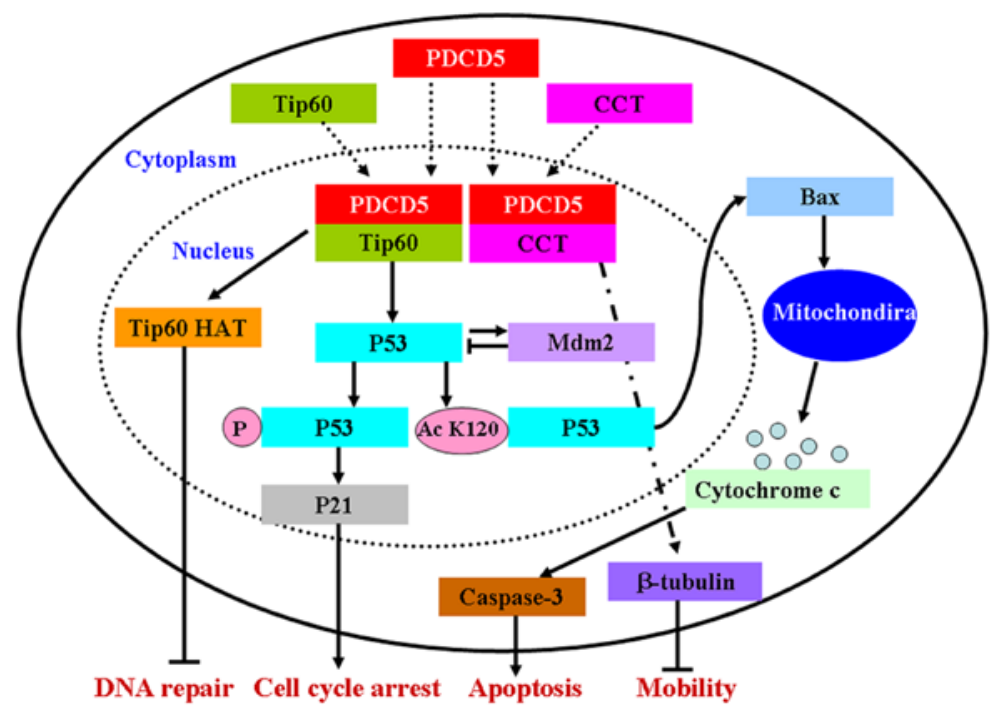

Figure 5. Schematic representation of the antitumor mechanisms of PDCD5 in cancer cells.

PDCD5 could counteract adriamycin resistance of osteosarcoma cells (Saos-2) by inhibiting P-glycoprotein (Pgp) expression. In the study of Han et al (29), they confirmed that various PDCD5 fragments [integrated (1-125 aa), truncated 1 (1-101 aa), and truncated 2 (41-125 aa)] could inhibit proliferation, and induce apoptosis and cell cycle arrest in the osteosarcoma cell line MG-63, and these effects were related to suppression of the Ras/Raf/MEK/ERK signaling pathway. This is an interesting result which showed that PDCD5 could induce apoptosis independently. Chondrosarcoma is the second most common primary malignant bone tumor after osteosarcoma (31). Compared with matched normal bone tissues, Chen et al (32) found that the levels of PDCD5 mRNA and protein were significantly decreased in chondrosarcoma tissues. PDCD5 was an independent prognostic factor in chondrosarcoma (32). In the following study, Chen et al (33) found that rhPDCD5 protein sensitizes chondrosarcomas to cisplatin chemotherapy in vitro and in vivo. These results indicated that PDCD5 may be used as a molecular marker in the diagnosis and prognosis of bone tumors.

Thoracic malignancy. The rs 1862214 polymorphism in $P D C D 5$ is predictive for lung cancer risk and prognosis, and that $P D C D 5$ may represent a novel tumor suppressor gene influencing lung cancer (33). However, in the study of Nanba et al (34), they found that the rs1862214 SNP of PDCD5 in Japanese population is not related to the risk of lung cancer. These results suggested the different roles of $P D C D 5$ in the pathogenesis of lung cancer in different ethnicity. The levels of PDCD5 mRNA and protein were also significantly decreased in laryngeal squamous cell carcinoma, indicating that PDCD5 may have an important role in the pathogenesis and development of laryngeal squamous cell carcinoma (35). Wang et al (36) confirmed that rhPDCD5 protein enhances the paclitaxel sensitivity of breast cancer in vitro and in vivo.

Abdominal tumor. Fu et al (9) demonstrated that PDCD5 expression is correlated with clinicopathological features and it may be a useful predictor of prognosis in patients with hepa- tocellular carcinoma after surgical resection. A recent study showed that PDCD5 could increase cisplatin sensitivity and decrease invasion in hepatic cancer cells (37). Compared with matched normal tissues, gastric cancer tissues contained lower level of PDCD5 protein (6). The survival rate of the patients with gastric cancer was dependent upon the expression level of PDCD5 (6). Xu et al (38) also found that PDCD5 sensitizes gastric cancer cells to cisplatin-induced apoptosis. Another study showed that combination of PDCD5 and cisplatin enhances apoptosis in colorectal cancer cells by activating the mitochondrial signaling pathway (39). A recent study of our team indicated that serum levels of PDCD5 protein were related with circulating $\mathrm{CD} 133^{+}$cells in gastric cancer patients (40).

Other tumors. Human glioma is one of the most frequent primary tumors of the central nervous system (7). A reduced expression of PDCD5 mRNA was found in glioma compared with normal brain tissue (41). Li et al (42) confirmed that PDCD5 promotes cisplatin-induced apoptosis in glioma cells (U87, U251 and T98G) by activating mitochondrial apoptotic pathway. PDCD5 mRNA and protein expression were downregulated in ovarian (43) and prostate cancer (44). rhPDCD5 protein increases doxorubicin-induced apoptosis in ovarian cancer SKOV3 cells (44). Notably, a recent study of Gao et al (45) confirmed that reduced PDCD5 protein is correlated with the degree of tumor differentiation in endometrioid endometrial carcinoma.

\section{The antitumor mechanisms of PDCD5}

Chen et al (46) found that PDCD5 exerts its effects through the pathway of mitochondria by modulating Bax translocation, cytochrome $c$ release and caspase-3 activation. In the study of Xu et al (47), it was found that PDCD5 may play a dual role in the Tip60 pathway: under normal growth conditions, PDCD5 contributes to maintaining a basal pool of Tip60 and histone acetyltransferase (HAT) activity; after DNA damage, PDCD5 functions as a Tip60 
coactivator to promote apoptosis through the Tip60-p53 pathway. Zhuge et al (48) found that PDCD5 interactions with Tip60 can accelerate DNA damage-induced apoptosis. A recent study showed that PDCD5 interacts with p53 and functions as a positive regulator in the p53 pathway (11). PDCD5 prevents ubiquitination of p53 in the cytoplasm by sequestering murine double minute $2(\mathrm{Mdm} 2)$, thereby increasing p53 stability (49). Genetic deletion of PDCD5 abrogates etoposide-induced p53 stabilization in gastric cancer cells (50). Furthermore, Park et al (51) found that PDCD5 knockdown greatly attenuated the effect of OTU deubiquitinase 5 (OTUD5) on p53 activation. These results indicated an essential role of PDCD5 in p53 activation.

Tracy et al (52) found that PDCD5 interacts with the cytosolic chaperonin containing tailless complex polypeptide 1 (CCT). PDCD5-CCT complex could exert its apoptotic function, at least in part through inhibition of $\beta$-tubulin folding (52). In the study of $\mathrm{Fu}$ et al (53), it was found that recombinant human PDCD5 exhibits an antitumor role in hepatocellular carcinoma cells via clathrin-dependent endocytosis.

PDCD5 inhibits the proliferation of cancer cells by the mitochondrial apoptotic pathway and the NF- $\mathrm{B}$ pathway $(54,55)$.

Collectively, these studies provide compelling evidence of the antitumor mechanisms of PDCD5. Better understanding of these mechanisms could create novel therapeutic opportunities in treating cancer cells (Fig. 5).

\section{Future perspectives}

To date, cancer is still a fatal disease, and relapse rates in patients remain high regardless of the intensity of treatment. In recent years, PDCD5 has received increased attention in the molecular biology of cancer. Based on previous results and concepts, PDCD5 could be used as a prognostic marker for cancers. We believe that these data will open new perspectives for a better understanding of PDCD5. However, we are only at the beginning of understanding the precise roles of PDCD5 in cancer cells. Current results did not have sufficient certainty to permit their use in cancer patients. Thus, PDCD5 safety and efficacy in cancer treatment may be significant issues in future studies.

\section{Acknowledgements}

The present review was supported by the Natural Scientific Foundation of Liaoning Province (2014021036), the General Project Scientific Research from the Department of Education of Liaoning Province (L2012288) and the Natural Scientific Foundation of China (81502107).

\section{References}

1. Liu H, Wang Y, Zhang Y, Song Q, Di C, Chen G, Tang J and Ma D: TFAR19, a novel apoptosis-related gene cloned from human leukemia cell line TF-1, could enhance apoptosis of some tumor cells induced by growth factor withdrawal. Biochem Biophys Res Commun 254: 203-210, 1999.

2. Spinola M, Meyer P, Kammerer S, Falvella FS, Boettger MB, Hoyal CR, Pignatiello C, Fischer R, Roth RB, Pastorino U, et al: Association of the PDCD5 locus with lung cancer risk and prognosis in smokers. J Clin Oncol 24: 1672-1678, 2006.
3. Liu D, Feng Y, Cheng Y and Wang J: Human programmed cell death 5 protein has a helical-core and two dissociated structural regions. Biochem Biophys Res Commun 318: 391-396, 2004.

4. Chen CH, Jiang Z, Yan JH, Yang L, Wang K, Chen YY, Han JY, Zhang JH and Zhou CM: The involvement of programmed cell death 5 (PDCD5) in the regulation of apoptosis in cerebral ischemia/reperfusion injury. CNS Neurosci Ther 19: 566-576, 2013.

5. Hedenfalk I, Duggan D, Chen Y, Radmacher M, Bittner M, Simon R, Meltzer P, Gusterson B, Esteller M, Kallioniemi OP, et al: Gene-expression profiles in hereditary breast cancer. $\mathrm{N}$ Engl J Med 344: 539-548, 2001.

6. Yang YH, Zhao M, Li WM, Lu YY, Chen YY, Kang B and Lu YY: Expression of programmed cell death 5 gene involves in regulation of apoptosis in gastric tumor cells. Apoptosis 11: 993-1001, 2006.

7. Li H, Wang Q, Gao F, Zhu F, Wang X, Zhou C, Liu C, Chen Y, Ma C, Sun W, et al: Reduced expression of PDCD5 is associated with high-grade astrocytic gliomas. Oncol Rep 20: 573-579, 2008.

8. Ruan GR, Qin YZ, Chen SS, Li JL, Ma X, Chang Y, Wang YZ, Fu JY and Liu YR: Abnormal expression of the programmed cell death 5 gene in acute and chronic myeloid leukemia. Leuk Res 30: 1159-1165, 2006.

9. Fu DZ, Cheng Y, He H, Liu HY and Liu YF: PDCD5 expression predicts a favorable outcome in patients with hepatocellular carcinoma. Int J Oncol 43: 821-830, 2013.

10. Wang Y, Li X, Wang L, Ding P, Zhang Y, Han W and Ma D: An alternative form of paraptosis-like cell death, triggered by TAJ/ TROY and enhanced by PDCD5 overexpression. J Cell Sci 117: $1525-1532,2004$

11. Xu L, Hu J, Zhao Y, Hu J, Xiao J, Wang Y, Ma D and Chen Y: PDCD5 interacts with $\mathrm{p} 53$ and functions as a positive regulator in the p53 pathway. Apoptosis 17: 1235-1245, 2012.

12. Xiao J, Liu C, Li G, Peng S, Hu J, Qu L, Lv P, Zhang Y, Ma D and Chen Y: PDCD5 negatively regulates autoimmunity by upregulating $\mathrm{FOXP}^{+}$regulatory T cells and suppressing Th17 and Th1 responses. J Autoimmun 47: 34-44, 2013.

13. Li K, Zhou Z, Wang YO, Liu J, Zhao HB, Yang J and Wang SQ: Pretreatment of mice with oligonucleotide prop5 protects them from influenza virus infections. Viruses 6: 573-581, 2014.

14. Liu D, Yao H, Chen Y, Feng Y, Chen Y and Wang J: The N-terminal 26-residue fragment of human programmed cell death 5 protein can form a stable alpha-helix having unique electrostatic potential character. Biochem J 392: 47-54, 2005.

15. Colin S, Jeanny JC, Mascarelli F, Vienet R, Al-Mahmood S, Courtois $\mathrm{Y}$ and Labarre J: In vivo involvement of heparan sulfate proteoglycan in the bioavailability, internalization, and catabolism of exogenous basic fibroblast growth factor. Mol Pharmacol 55: 74-82, 1999.

16. Chen Y, Sun R, Han W, Zhang Y, Song Q, Di C and Ma D: Nuclear translocation of PDCD5 (TFAR19): An early signal for apoptosis? FEBS Lett 509: 191-196, 2001.

17. Johnson VL and Hunter DJ: The epidemiology of osteoarthritis. Best Pract Res Clin Rheumatol 28: 5-15, 2014.

18. Loeser RF: Aging and osteoarthritis: The role of chondrocyte senescence and aging changes in the cartilage matrix. Osteoarthritis Cartilage 17: 971-979, 2009.

19. Cheng AX, Lou SQ, Zhou HW, Wang Y and Ma DL: Expression of PDCD5, a novel apoptosis related protein, in human osteoarthritic cartilage. Acta Pharmacol Sin 25: 685-690, 2004.

20. Yi C, Ma C, Xie Z, Zhang G, Song W, Zhou X and Cao Y: Downregulation of programmed cell death 5 by insulin-like growth factor 1 in osteoarthritis chondrocytes. Int Orthop 37: 937-943, 2013.

21. Firestein GS: Evolving concepts of rheumatoid arthritis. Nature 423: 356-361, 2003.

22. Wang N, Lu HS, Guan ZP, Sun TZ, Chen YY, Ruan GR, Chen ZK, Jiang $J$ and Bai CJ: Involvement of PDCD5 in the regulation of apoptosis in fibroblast-like synoviocytes of rheumatoid arthritis. Apoptosis 12: 1433-1441, 2007.

23. Wang J, Guan Z and Ge Z: Plasma and synovial fluid programmed cell death 5 (PDCD5) levels are inversely associated with TNF- $\alpha$ and disease activity in patients with rheumatoid arthritis. Biomarkers 18: 155-159, 2013.

24. Wang JF, Guan ZP, Zhang SL, Pei Z, Chen YY and Pan H: Programmed cell death 5 correlates with disease activity and interleukin-17 in serum and synovial fluid of rheumatoid arthritis patients. Chin Med J (Engl) 126: 296-299, 2013. 
25. Xiao J, Liu W, Chen Y and Deng W: Recombinant human PDCD5 (rhPDCD5) protein is protective in a mouse model of multiple sclerosis. J Neuroinflammation 12: 117, 2015.

26. Ruan GR, Zhao HS, Chang Y, Li JL, Qin YZ, Liu YR, Chen SS and Huang XJ: Adenovirus-mediated PDCD5 gene transfer sensitizes K562 cells to apoptosis induced by idarubicin in vitro and in vivo. Apoptosis 13: 641-648, 2008.

27. Shi L, Song Q, Zhang Y, Lou Y, Wang Y, Tian L, Zheng Y, Ma D, $\mathrm{Ke} \mathrm{X}$ and Wang Y: Potent antitumor activities of recombinant human PDCD5 protein in combination with chemotherapy drugs in K562 cells. Biochem Biophys Res Commun 396: 224-230, 2010.

28. Thompson RC Jr, Cheng EY, Clohisy DR, Perentesis J, Manivel C and Le CT: Results of treatment for metastatic osteosarcoma with neoadjuvant chemotherapy and surgery. Clin Orthop Relat Res 397: 240-247, 2002.

29. Han XR, Sun Y and Bai XZ: The anti-tumor role and mechanism of integrated and truncated PDCD5 proteins in osteosarcoma cells. Cell Signal 24: 1713-1721, 2012.

30. Zhao H, Peng C, Ruan G, Zhou J, Li Y and Hai Y: Adenovirusdelivered PDCD5 counteracts adriamycin resistance of osteosarcoma cells through enhancing apoptosis and inhibiting Pgp. Int J Clin Exp Med 7: 5429-5436, 2014.

31. Rozeman LB, Cleton-Jansen AM and Hogendoorn PC: Pathology of primary malignant bone and cartilage tumours. Int Orthop 30: 437-444, 2006

32. Chen C, Zhou H, Xu L, Liu X, Liu Z, Ma D, Chen Y and Ma Q: Prognostic significance of downregulated expression of programmed cell death 5 in chondrosarcoma. J Surg Oncol 102: 838-843, 2010.

33. Chen C, Zhou H, Xu L, Xu D, Wang Y, Zhang Y, Liu X, Liu Z, Ma D, Ma Q, et al: Recombinant human PDCD5 sensitizes chondrosarcomas to cisplatin chemotherapy in vitro and in vivo. Apoptosis 15: 805-813, 2010

34. Nanba K, Toyooka S, Soh J, Tsukuda K, Yamamoto H, Sakai A, Ouchida M, Kobayashi N, Matsuo K, Koide N, et al: The allelic distribution of a single nucleotide polymorphism in the PDCD5 gene locus of Japanese non-small cell lung cancer patients. Mol Med Rep 1: 667-671, 2008.

35. Xu F, Wu K, Zhao M, Qin Y and Xia M: Expression and clinical significance of the programmed cell death 5 gene and protein in laryngeal squamous cell carcinoma. J Int Med Res 41: 1838-1847, 2013.

36. Wang L, Wang C, Su B, Song Q, Zhang Y, Luo Y, Li Q, Tan W, Ma D and Wang L: Recombinant human PDCD5 protein enhances chemosensitivity of breast cancer in vitro and in vivo. Biochem Cell Biol 91: 526-531, 2013.

37. Fan GL, Yao Y, Yao L and Li Y: PDCD5 transfection increases cisplatin sensitivity and decreases invasion in hepatic cancer cells. Oncol Lett 9: 411-417, 2015.

38. Xu HY, Chen ZW, Pan YM,Fan L, Guan J and Lu YY: Transfection of PDCD5 effect on the biological behavior of tumor cells and sensitized gastric cancer cells to cisplatin-induced apoptosis. Dig Dis Sci 57: 1847-1856, 2012.

39. Yin A, Jiang Y, Zhang X, Zhao $\mathrm{J}$ and Luo $\mathrm{H}$ : Transfection of PDCD5 sensitizes colorectal cancer cells to cisplatin-induced apoptosis in vitro and in vivo. Eur J Pharmacol 649: 120-126, 2010.

40. Wang D, Wang W, Song CL and Xia P: The roles of serum PDCD5 in circulating CD133 positive cells of the patients with gastric cancer. Tumour Biol: Mar 31, 2016 (Epub ahead of print].
41. Olischar M, Stavroudis T, Karp JK, Kaufmann WE and Theda C Medical and ethical challenges in the case of a prenatally undiagnosed massive congenital brain tumor. J Perinatol 35: 773-775, 2015.

42. Li H, Zhang X, Song X, Zhu F, Wang Q, Guo C, Liu C, Shi Y, Ma C, Wang X, et al: PDCD5 promotes cisplatin-induced apoptosis of glioma cells via activating mitochondrial apoptotic pathway. Cancer Biol Ther 13: 822-830, 2012.

43. Gao L, Ye X, Ma RQ, Cheng HY, Han HJ, Cui H, Wei LH and Chang XH: Low programmed cell death 5 expression is a prognostic factor in ovarian cancer. Chin Med J (Engl) 128: 1084-1090, 2015.

44. Du YJ, Xiong L, Lou Y, Tan WL and Zheng SB: Reduced expression of programmed cell death 5 protein in tissue of human prostate cancer. Chin Med Sci J 24: 241-245, 2009.

45. Gao M, Gao W, Wang Z, Liu Y, Li Y, Wei C, Sun Y, Guo C, Zhang L, Wei Z, et al: The reduced PDCD5 protein is correlated with the degree of tumor differentiation in endometrioid endometrial carcinoma. Springerplus 5: 988, 2016.

46. Chen LN, Wang Y, Ma DL and Chen YY: Short interfering RNA against the PDCD5 attenuates cell apoptosis and caspase-3 activity induced by Bax overexpression. Apoptosis 11: 101-111, 2006.

47. Xu L, Chen Y, Song Q, Xu D, Wang Y and Ma D: PDCD5 interacts with Tip60 and functions as a cooperator in acetyltransferase activity and DNA damage-induced apoptosis. Neoplasia 11: 345-354, 2009.

48. Zhuge C, Chang Y, Li Y, Chen Y and Lei J: PDCD5-regulated cell fate decision after ultraviolet-irradiation-induced DNA damage. Biophys J 101: 2582-2591, 2011.

49. Essers PB, Klasson TD, Pereboom TC, Mans DA, Nicastro M, Boldt K, Giles RH and MacInnes AW: The von Hippel-Lindau tumor suppressor regulates programmed cell death 5-mediated degradation of Mdm2. Oncogene 34: 771-779, 2015.

50. Choi HK, Choi Y, Park ES, Park SY, Lee SH, Seo J, Jeong MH, Jeong JW, Jeong JH, Lee PC, et al: Programmed cell death 5 mediates HDAC 3 decay to promote genotoxic stress response. Nat Commun 6: 7390, 2015.

51. Park SY, Choi HK, Choi Y, Kwak S, Choi KC and Yoon HG: Deubiquitinase OTUD5 mediates the sequential activation of PDCD5 and p53 in response to genotoxic stress. Cancer Lett 357: 419-427, 2015.

52. Tracy CM, Gray AJ, Cuéllar J, Shaw TS, Howlett AC, Taylor RM, Prince JT, Ahn NG, Valpuesta JM and Willardson BM: Programmed cell death protein 5 interacts with the cytosolic chaperonin containing tailless complex polypeptide 1 (CCT) to regulate $\beta$-tubulin folding. J Biol Chem 289: 4490-4502, 2014.

53. Fu DZ, Cheng Y, He H, Liu HY and Liu YF: Recombinant human PDCD5 exhibits an antitumor role in hepatocellular carcinoma cells via clathrin-dependent endocytosis. Mol Med Rep 12: 8135-8140, 2015.

54. Xu S, Sui G, Yuan L and Zou Z: Expression of programmed cell death 5 protein inhibits progression of lung carcinoma in vitro and in vivo via the mitochondrial apoptotic pathway. Mol Med Rep 10: 2059-2064, 2014.

55. Murshed F, Farhana L, Dawson MI and Fontana JA: NF-кB p65 recruited SHP regulates PDCD5-mediated apoptosis in cancer cells. Apoptosis 19: 506-517, 2014. 